\title{
Dynamic Nestin expression during hair follicle maturation and the normal hair cycle
}

\author{
RUOSI CHEN, YONG MIAO and ZHIQI HU \\ Department of Plastic and Cosmetic Surgery, Nanfang Hospital, Guangzhou, Guangdong 510515, P.R. China
}

Received March 13, 2017; Accepted September 3, 2018

DOI: $10.3892 / \mathrm{mmr} .2018 .9691$

\begin{abstract}
Nestin, a type-VI intermediate filament protein, serves as a marker for neural stem cells, and is also known to be expressed in follicle stem cells. Hair follicles go through repeated cycles of anagen (growth), catagen (regression) and telogen (quiescence) throughout the life of mammals following morphogenesis. In the present study it was demonstrated that in mice, the maturation of hair follicles includes the period between morphogenesis and the first anagen (4 weeks of age). Skin samples from Nestin-green fluorescent protein (GFP) ${ }^{+}$ mice at different hair follicle stages were collected, and immunostaining for Nestin and Ki67 was performed. It was identified that during morphogenesis, Nestin-GFP expression was rarely detected and it gradually increased during maturation (0-4 weeks) in hair follicle dermal cells. In mature hair follicle dermal cells, Nestin and the proliferation marker Ki67 were highly expressed in anagen, while during telogen, they were markedly decreased. Additionally, lineage tracing data demonstrated that peri-follicular Nestin ${ }^{+}$cells during morphogenesis differentiated into cluster of differentiation $31^{+}$cells.
\end{abstract}

\section{Introduction}

As the primary barrier to the body, the skin protects against dehydration, mechanical injury and microbial infection (1). Skin is composed of an outer epidermis and the underlying dermis separated by a basement membrane. Hair follicles are highly sensitive mini-organs comprising epidermal keratinocytes and mesenchymal compartments (2-4). Hair growth is a cyclic regeneration phenomenon regulated by connections between epithelial and dermal compartments (5-8). Hair follicles go through repeated cycles of anagen (growth), catagen (regression) and telogen (quiescence) throughout the life of mammals (9-12). Prior to the start of each cycle there is a phase of hair follicle morphogenesis. Hair follicle morphogenesis and

Correspondence to: Dr Zhiqi Hu, Department of Plastic and Cosmetic Surgery, Nanfang Hospital, 1838 North Guangzhou Avenue, Baiyun, Guangzhou, Guangdong 510515, P.R. China

E-mail: doctor_zhiqihu@hotmail.com

Key words: nestin, hair follicle, hair cycle the subsequent hair phases follow a precise timescale (13). The period beginning with hair morphogenesis until the first hair cycle is considered to be the maturation of the hair follicle.

Nestin, originally discovered in neuroepithelial stem cells, is an intermediate filament protein expressed during the early stages of development $(1,14)$. Hair follicles contain a distinct population of follicular stem cells that express Nestin (15). Using Nestin-green fluorescent protein (GFP) transgenic mice, researchers demonstrated that during telogen and in early anagen, Nestin-GFP ${ }^{+}$cells are primarily in the bulge area $(9,16)$. However, in mid- and late anagen, the GFP-expressing cells are located in the upper outer-root sheath in addition to the bulge area (9). However, Nestin expression between morphogenesis and the postnatal regular hair cycle is not well studied. In the present study, it was demonstrated that during morphogenesis, Nestin-GFP expression was detected rarely, and gradually increased during maturation (0-4 weeks) in hair follicle dermal cells. In mature hair follicle dermal cells, Nestin and Ki67 were highly expressed in anagen, while during telogen, they were markedly decreased. Additionally, the lineage tracing data demonstrated that peri-follicular Nestin ${ }^{+}$cells during morphogenesis differentiated into vascular cells.

\section{Materials and methods}

Animals and treatment. Nestin-GFP mice were provided by Dr Grigori Enikolopov at Cold Spring Harbor Laboratory (Cold Spring Harbor, NY, USA). Nestin-Cre ${ }^{\text {ERT2 mice }}$ (stock no. 003771) and B6.129X1-Gt (ROSA) 26Sortm1 [enhanced yellow fluorescent protein (EYFP)] Cos/J mice (stock no. 006148) were purchased from Jackson Laboratory (Bar Harbor, ME, USA). Five male mice were used in each group. The mice were 8-days-old, 2-weeks-old, 4-weeks-old, 8-weeks-old and 12-weeks-old, whose average weight were 5, $7,12,19$ and $25 \mathrm{~g}$, respectively. For the lineage tracing experiment, mice were injected with tamoxifen $(80 \mathrm{mg} / \mathrm{kg})$ to induce Cre-ER activity 8 days (P8) following birth and tested at 4 weeks old. All animal experiments were performed under the approval of the Institutional Animal Care and Use Committee at Southern Medical University (Guangzhou, China). Mice were housed at the Department of Laboratory Animal Science, Southern Medical University (Guangzhou, China), and maintained under a 12-h light/dark cycle, an ambient temperature of $22 \pm 2^{\circ} \mathrm{C}$ and a constant humility of $60 \pm 10 \%$, with food and water provided ad libitum. 
Flow cytometry analysis. For flow cytometry analysis of Nestin-GFP' ${ }^{-}$and $\mathrm{GFP}^{+}$cells from the whole skin, the skin from Nestin-GFP mice was dissected. Following hair removal, the skin was lightly defatted before $2 \mathrm{ml}$ of Trypsin-EDTA (0.5\%, 10X; Thermo Fisher Scientific, Inc., Waltham, MA, USA) was added, followed by incubation at $4^{\circ} \mathrm{C}$ overnight. Dermis were separated and cut into small pieces, followed by digestion in protease solution $[2 \mathrm{mg} / \mathrm{ml}$ collagenase I and $2.5 \mathrm{mg} / \mathrm{ml}$ trypsin in phosphate-buffered saline (PBS) at $\left.37^{\circ} \mathrm{C}\right]$ for $1 \mathrm{~h}$. Cells within the supernatant were collected for flow cytometry. Following red blood cell lysis (in order to remove the hemocytes) with commercial ammonium chloride-potassium lysis buffer (Quality Biological, Inc., Gaithersburg, MD, USA), the cells were analyzed according to CD45 and GFP expression. Flow cytometry analysis was performed using a FACSCalibur flow cytometer and CellQuest software (version 5.1, Becton-Dickinson Biosciences). The primary antibodies used were FITC-conjugated anti-mouse GFP (cat. no. 338008; 1:200; BioLegend, Inc., San Diego, CA, USA), PerCP-conjugated anti-mouse CD45 (cat. no. 103130; 1:200; BioLegend, Inc.). Briefly, dermal cells were blocked with $1 \%$ BSA (cat. no. 05470; Sigma-Aldrich; Merck KGaA, Darmstadt, Germany) for 30 min on ice and following washing with PBS, the primary antibodies were added and incubated on ice for $15 \mathrm{~min}$.

Immunofluorescence. Following sacrifice, the skin of the mice was resected and fixed in $4 \%$ ice-cold paraformaldehyde solution for $1 \mathrm{~h}$ and decalcified by immersion in $30 \%$ sucrose for $24 \mathrm{~h}$. Finally, tissues were embedded in optimal cutting temperature compound (Sakura Finetek USA, Inc, Torrance, CA, USA). Sections of skin (10- $\mu \mathrm{m}$ thick) were harvested for immunofluorescence staining.

For the staining, the sections were incubated with the following primary antibodies: Mouse Ki67 (cat. no. ab15580; 1:200), GFP (cat. no. ab290; 1:200) and cluster of differentiation (CD)31 (cat. no. ab28364; 1:50) (all from Abcam, Cambridge, UK). Slides were rinsed with TBST (cat. no. T5912; Sigma-Aldrich; Merck KGaA), blocked with 3\% BSA (cat. no. 05470; Sigma-Aldrich; Merck KGaA) for $1 \mathrm{~h}$ at room temperature and then incubated with the primary antibody overnight at $4^{\circ} \mathrm{C}$, followed by incubation with FITC or Cy3-conjugated secondary antibodies (FITC-conjugated secondary antibodies: cat. no. 711-546-152; 1:1,000; Cy3-conjugated secondary antibodies: cat. no. 711-167-003; 1:1,000; Jackson ImmunoResearch Europe, Ltd., Newmarket $\mathrm{UK}$ ) for $1 \mathrm{~h}$ at room temperature in the dark. Nuclei were counterstained with 4',6-diamidino-2-phenylindole (Sigma-Aldrich; Merck KGaA). The sections were mounted with the ProLong Antifade kit (Molecular Probes; Thermo Fisher Scientific, Inc.) and were observed under a Zeiss LSM780 confocal microscope (Zeiss AG, Oberkochen, Germany).

Statistical analysis. Data are presented as the mean \pm standard deviation of 3 independent experiments. One-way analysis of variance followed by the Bonferroni post hoc test was applied. All data were normally distributed and had similar variation between groups. Statistical analysis was performed using SAS version 9.3 software (SAS Institute, Inc., Cary, NC, USA).
$\mathrm{P}<0.05$ was considered to indicate a statistically significant difference.

\section{Results}

Maturation of hair follicle dermal cells is characterized by gain-of-nestin expression. Nestin is required for the self-renewal, proliferation and cell cycle progression of hair follicle cells (17-19). The mouse hair cycle follows a precise time scale. A gradual induction of Nestin-GFP signaling was detected in male Nestin- $\mathrm{GFP}^{+}$mice in hair follicle dermal cells at P8, and in 2- and 4-week-old mice (Fig. 1A and B). According to the immunofluorescence staining results, Nestin-GFP was expressed at low levels at P8 (morphogenesis) in hair follicle dermal cells and progressively increased at 2 and 4 weeks of age (first catagen and anagen; Fig. 1A and B). Therefore, it appeared that the period spanning morphogenesis until the first anagen represented the maturation of hair follicle and, following this, hair follicles follow a regular hair cycle.

Mature hair follicle dermal cells co-express nestin and Ki67 during anagen. Given the induction of Nestin-GFP signaling in male Nestin-GFP mice during maturation, the proliferation capacity of hair follicle dermal cells during this period was investigated by immunofluorescence staining with the Ki67 proliferation marker. The high amount of $\mathrm{Ki}^{+} 7^{+}$dermal cells observed at P8 (morphogenesis) indicated that the cells have high proliferative activity during morphogenesis. Ki67 expression was substantially maintained at 2 weeks of age, when the first catagen begins, and increased at 4 weeks, when first anagen begins (Fig. 1C and D). Notably, co-immunofluorescence staining of Ki67 and Nestin-GFP at different time points indicated that at $\mathrm{P} 8$, few $\mathrm{Ki}^{+} 7^{+}$hair follicle dermal cells also expressed Nestin-GFP. At 2 weeks, nearly $20 \%$ of $\mathrm{Ki}^{+} 7^{+}$hair follicle dermal cells expressed Nestin-GFP (Fig. 1E and F). In 4-week-old mice, nearly all $\mathrm{Ki} 7^{+}$hair follicle dermal cells expressed Nestin-GFP, indicating that mature Nestin ${ }^{+}$hair follicle dermal cells are of high proliferative capacity during the first anagen (Fig. 1E and F). During the maturation of hair follicles, hair follicle dermal cells begin expressing Nestin, and mature cells are of high proliferative capacity.

Dynamic nestin expression during the normal hair cycle in hair follicle dermal cells. Whether Nestin is highly expressed during the normal hair cycle was also investigated. To determine this, skin from 4-, 8- and 12-week-old male Nestin-GFP ${ }^{+}$ mice was collected, corresponding to the first anagen, second telogen and second anagen of the murine hair cycle, respectively. Nestin-GFP was highly expressed in anagen (growing phase; 4 and 12 weeks) and significantly decreased in telogen (quiescent phase; 8 weeks; Fig. 2A and B).

Nestin may serve as marker of high proliferation during the normal hair cycle. To investigate Ki67 expression during the normal hair cycle in hair follicle dermal cells, Ki67 immunofluorescence staining was performed on 4-, 8- and 12-week-old male Nestin-GFP ${ }^{+}$mice. High Ki67 expression levels were observed at 4 and 12 weeks, representing the first and second anagen of the murine hair cycle, respectively, indicating that the cells were in a highly proliferative state. At 8 weeks, when 

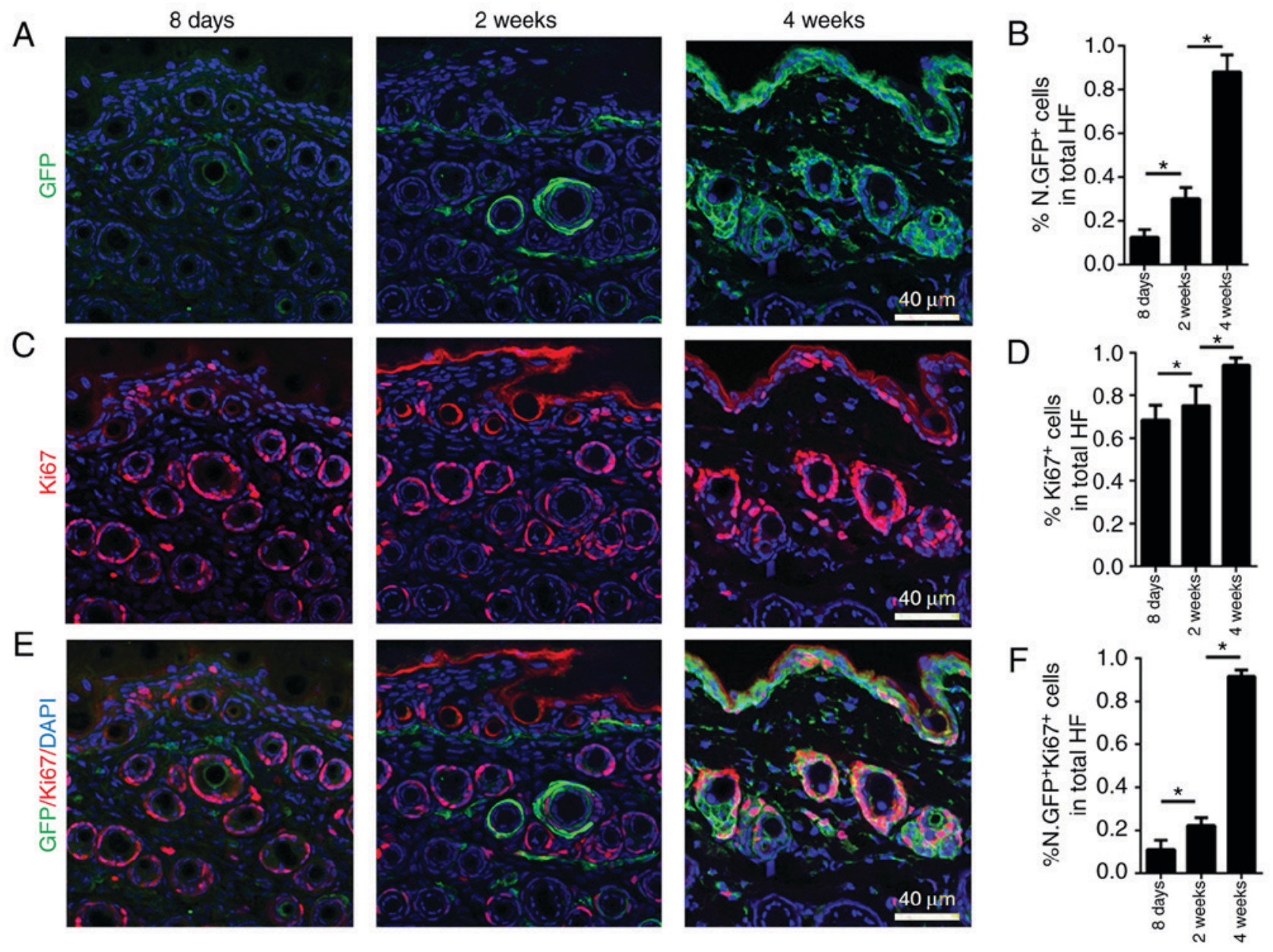

Figure 1. HF dermal cells in the dermis layer exhibit Nestin expression during hair morphogenesis. Representative images of (A) immunofluorescence staining and (B) quantitative analysis of $\mathrm{GFP}^{+}$cells, (C) immunofluorescence staining and (D) quantification of Ki67 ${ }^{+}$cells, and (E) immunofluorescence staining and (F) quantification of $\mathrm{GFP}^{+} / \mathrm{Ki}^{+}$cells, in the dermis layer from P8, 2- and 4-week-old male Nestin-GFP ${ }^{+}$mice (n=5). GFP is in green, Ki67 in red and DAPI stains the nuclei blue. GFP, green fluorescent protein; DAPI, 4',6-diamidino-2-phenylindole; HF, hair follicle. ${ }^{*} \mathrm{P}<0.05$.

mouse hair follicles were in telogen (quiescent phase), Ki67 expression levels were significantly decreased (Fig. 2C and D). Co-immunofluorescence staining of Ki67 and Nestin-GFP indicated that nearly all $\mathrm{Ki}^{+} 7^{+}$cells were also Nestin-GFP ${ }^{+}$, and the expression of the two markers was increased in anagen and decreased in telogen (Fig. 2E and F). The fact that Nestin had the same expression pattern as Ki67 during the normal hair cycle in hair follicle dermal cells indicated that Nestin may serve as a marker of high proliferation during the normal hair cycle. Furthermore, flow cytometry analysis of the isolated cells demonstrated that the percentage of $\mathrm{GFP}^{+}$ cells was gradually increased in mice of 2 and 4 weeks of age compared with those at 8 days of age, which was in accordance with the immunofluorescence staining results (Fig. 3A and B). Additionally, flow cytometry analysis of the isolated cells demonstrated that the percentage of $\mathrm{GFP}^{+}$cells was increased during anagen (12 weeks) and decreased during telogen $(8$ weeks; Fig. 3C and D).

Nestin positive cells also express CD31. At P8, when morphogenesis occurs, Nestin-GFP was rarely expressed in hair follicle dermal cells. However, Nestin-GFP ${ }^{+}$cells were detected in the peri-follicular area (Fig. 1A). The lineage fate of the Nestin ${ }^{+}$ cells was traced at P8 using Nestin-Cre::ROSA26-EYFP mice. Examination of the fate of Nestin-EYFP ${ }^{+}$cells 3 weeks post-tamoxifen injection revealed that $\sim 20 \%$ of the EYFP-labeled cells were also CD31+ (Fig. 4).

\section{Discussion}

In mice, the first two postnatal hair cycles are synchronized (13). Hair follicles are an ideal system for studying how stem cells interact with progeny in the niche between quiescence and regeneration (5). Murine hair follicles undergo a precise hair cycle, which follows a specific timescale following hair morphogenesis (13). From birth to 2 weeks of age, murine hair follicles are in a morphogenic phase, followed by 2-, 3- and 4-weeks of age when hair follicles undergo the first postnatal catagen, telogen and anagen, respectively. The second hair cycle occurs at 6-, 7- and 12-weeks of age, indicating the second catagen, telogen and anagen, respectively (13). Using this guide to classify hair phases, a previous study demonstrated that Nestin is expressed in different hair follicle locations in different developmental phases (9). However, Nestin expression between morphogenesis and the postnatal regular hair cycle is not well studied.

Nestin, a type-VI intermediate filament protein, serves as a marker for neural stem cells and is also known to be 

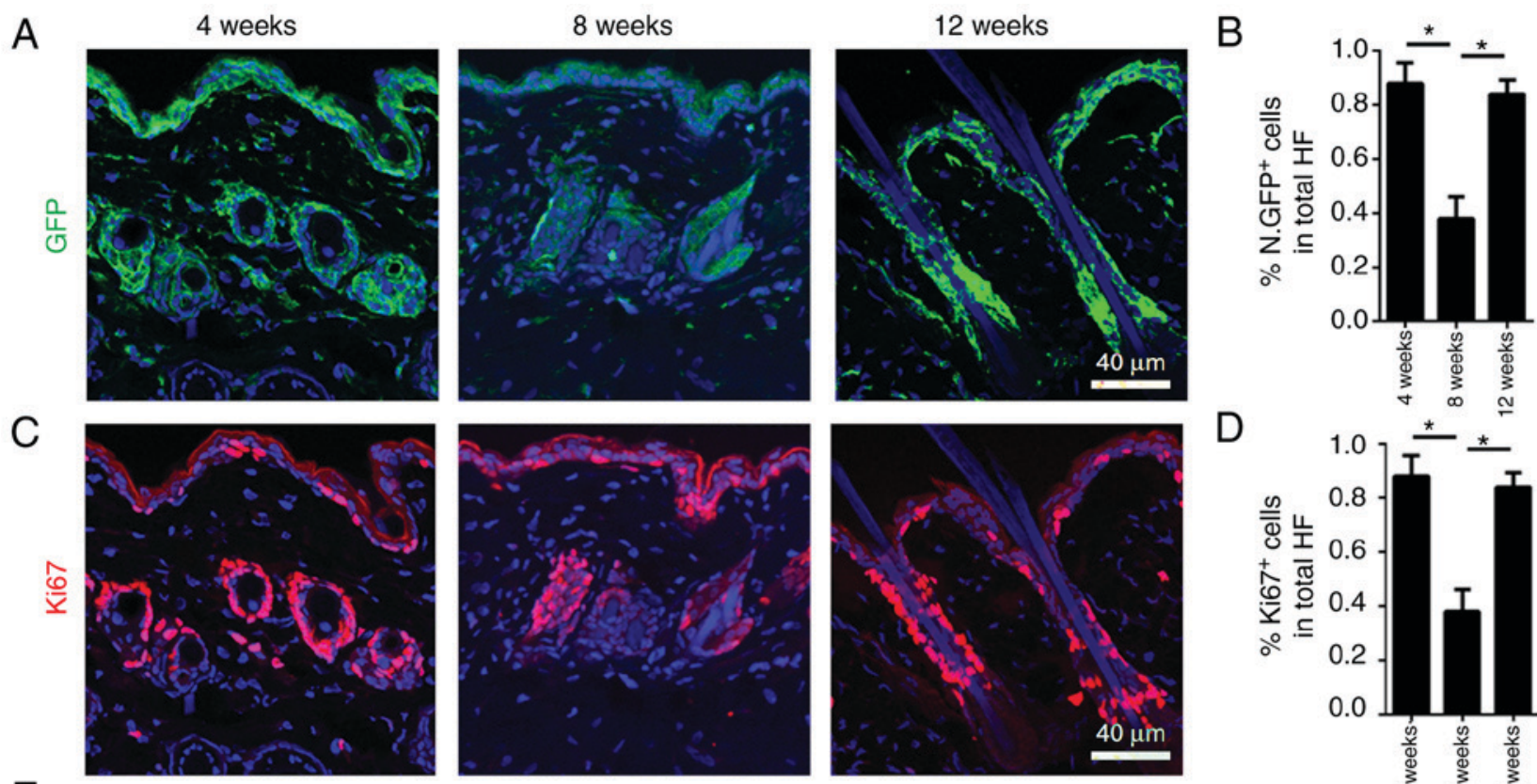

D

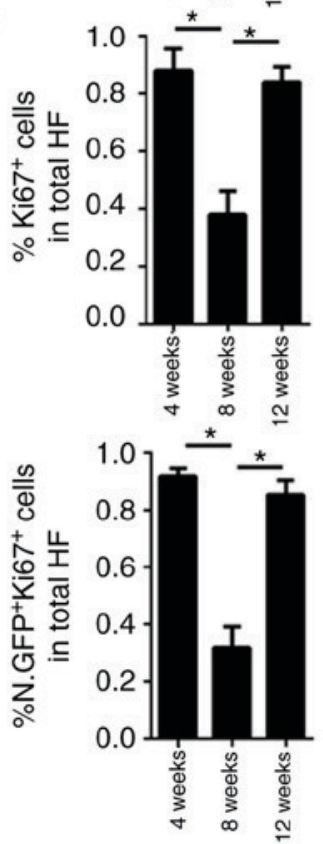

Figure 2. Dynamic Nestin expression in HF dermal cells during the hair cycle. Representative images of (A) immunofluorescence staining and (B) quantitative analysis of $\mathrm{GFP}^{+}$cells, (C) immunofluorescence staining and (D) quantification of Ki67 cells, and (E) immunofluorescence staining and (F) quantification of $\mathrm{GFP}^{+} / \mathrm{Ki}^{+} 7^{+} \mathrm{cells}$, in the dermis layer from 4-, 8- and 12-week-old male Nestin-GFP ${ }^{+}$mice $(\mathrm{n}=5)$. GFP is in green, Ki67 in red and DAPI stains the nuclei blue. GFP, green fluorescent protein; DAPI, 4',6-diamidino-2-phenylindole; HF, hair follicle. " $\mathrm{P}<0.05$.
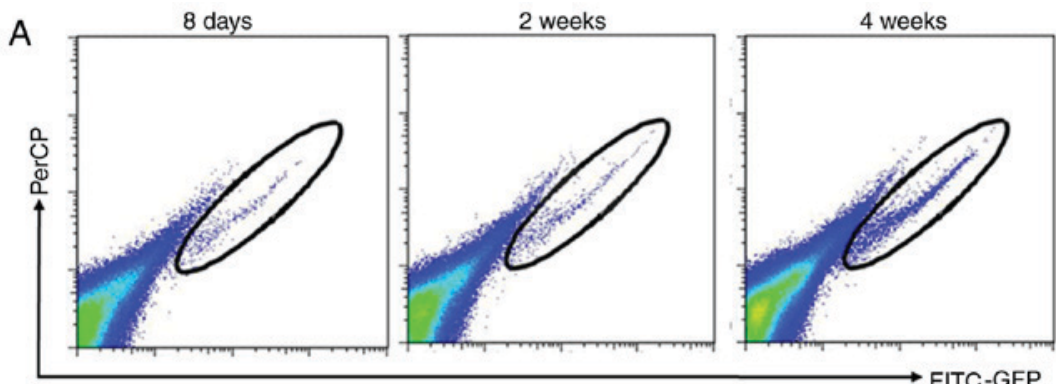

C

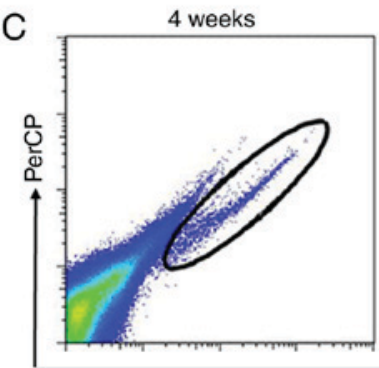

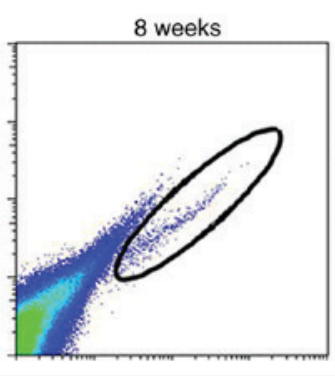

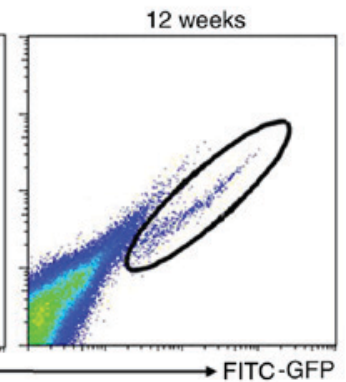

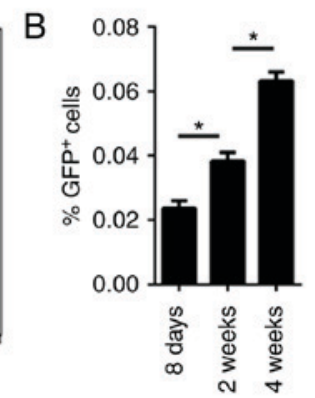

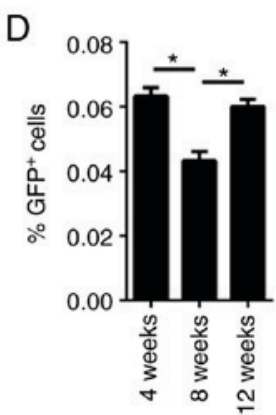

Figure 3. Flow cytometry analysis of dynamic Nestin expression in hair follicle dermal cells. Representative images of (A) flow cytometry analysis and (B) quantification of the $\mathrm{GFP}^{+}$cells in the skin of $\mathrm{P} 8,2$ - and 4-week-old mice, followed by (C) flow cytometry analysis and (D) quantification of the GFP ${ }^{+}$cells, in the skin of 4-, 8- and 12-week-old male Nestin-GFP mice. GFP, green fluorescent protein; FITC, fluorescein isothiocyanate. ${ }^{*} \mathrm{P}<0.05$. 


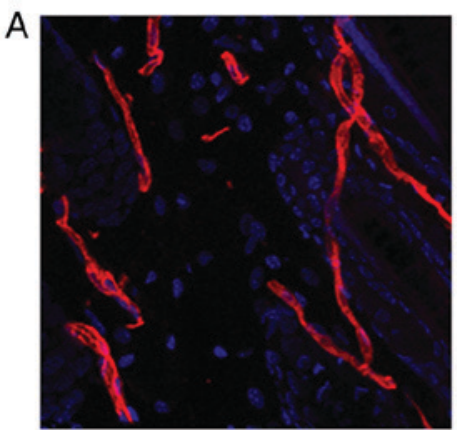

CD31/DAPI

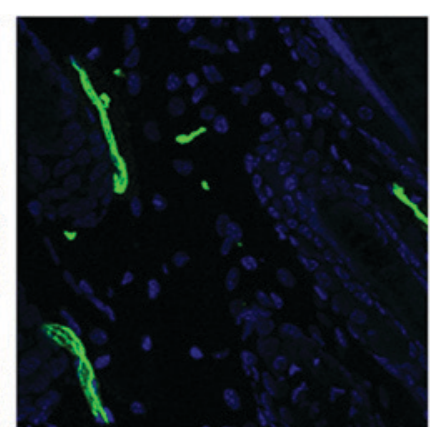

EYFP/DAPI

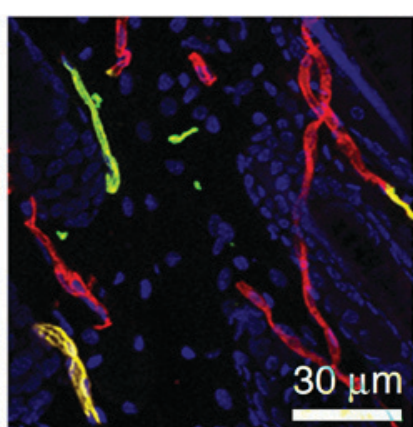

CD31/EYFP/DAPI

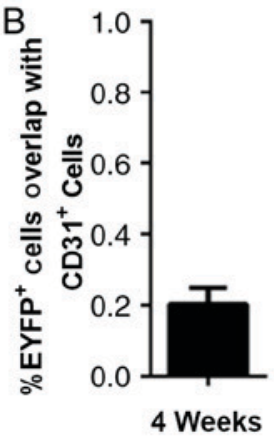

4 Weeks

Figure 4. Nestin ${ }^{+}$cells are involved in dermis layer CD31+ vessel formation during hair morphogenesis. Nestin-Cre::ROSA26-EYFP mice were given a single dose of tamoxifen at P8. Representative images of (A) immunofluorescence staining and (B) quantitative analysis of $\mathrm{CD}^{+} 1^{+} / \mathrm{EYFP}^{+}$cells in skin dermis layer sections from 4-week-old male Nestin-Cre::ROSA26-EYFP mice $(n=5)$. EYFP is presented in green, CD31 is red and DAPI stains the nuclei blue. DAPI, 4',6-diamidino-2-phenylindole; EYFP, enhanced yellow fluorescent protein; CD31, cluster of differentiation 31.

expressed in follicle stem cells (14). Previous studies have demonstrated that Nestin is required for the self-renewal, proliferation and cell cycle progression of the cells, particularly in neural progenitor cells (17-19). Consistent with all these findings, the present study demonstrated that during the hair follicle cycle, there was a high percentage of $\mathrm{Ki} 67^{+}$cells among the Nestin-expressing cells in the dermis layer at 4 and 12 weeks following birth, which represent the first and second anagen of the murine hair cycle, respectively. Notably, at 8 weeks of age, which is the first telogen (quiescent phase), $\mathrm{Ki} 67^{+} / \mathrm{Nestin}^{+}$hair follicle cell numbers decreased. These results indicated the high proliferative capacity of these cells, likely due to a high demand for hair follicle replacement during anagen and not during telogen. All these results suggested that Nestin ${ }^{+}$cells in the dermis layer proliferate more rapidly compared with Nestin ${ }^{-}$cells in the same region. A notable phenomenon from the present study is that during early hair follicle morphogenesis, Nestin-GFP ${ }^{+}$cells gradually appeared in the dermis layer until the first anagen, which indicated that Nestin expression may be a sign of hair follicle maturation.

Previous findings revealed that $\mathrm{Nestin}^{-\mathrm{Cre}^{+}}$and Nestin-GFP ${ }^{+}$cells are able to label endothelial, mesenchymal lineage and Schwann precursor cells (20-24). At P8, when morphogenesis occurs, Nestin-GFP was rarely expressed in hair follicle dermal cells. However, Nestin-GFP ${ }^{+}$cells were detected in the peri-follicular area. In the present study, a single dose of tamoxifen was administered to P8 Nestin-Cre ${ }^{\text {ERT2}}$; ROSA26-EYFP in order to preform lineage tracing, and skin samples were harvested after 3 weeks. It was illustrated that during hair follicle morphogenesis, peri-follicular Nestin ${ }^{+}$cells also expressed CD31, which is an endothelial cell marker. This finding may divide Nestin ${ }^{+}$ cells in the dermis layer into two different populations: Peri-follicular Nestin ${ }^{+}$cells during morphogenesis that are of endothelial lineage, and hair follicle Nestin ${ }^{+}$cells that are hair follicle precursor cells.

In conclusion, the expression of Nestin in hair follicles during morphogenesis and maturation was investigated. Additionally, Nestin may serve as a marker of high proliferation during the normal hair cycle, and was highly expressed during anagen and decreased during telogen in the murine hair cycle. Furthermore, certain $\mathrm{Nestin}^{+}$cells may serve a role in other processes, including angiogenesis during morphogenesis.

\section{Acknowledgements}

Not applicable.

\section{Funding}

The present study was supported by the Natural Science Foundation of China (grant nos. 81471900, 81701929 and 81772104) and the Natural Science Foundation of Guangdong Province (grant nos. 2015A030311001 and 2017A030310120) and the Science and Technology Planning Project of Guangzhou City (grant no. 201508020262).

\section{Availability of data and materials}

The datasets used and/or analyzed during the current study are available from the corresponding author on reasonable request.

\section{Authors' contributions}

RC performed the experiments and wrote the manuscript. YM analyzed the data. $\mathrm{ZH}$ designed the study and critically revised the manuscript for important intellectual content.

\section{Ethics approval and consent to participate}

All animal experiments were performed under the approval of the Institutional Animal Care and Use Committee at Southern Medical University (Guangzhou, China).

\section{Patient consent for publication}

Not applicable.

\section{Competing interests}

The authors declare that they have no competing interests. 


\section{References}

1. Falodah FA and Al-Karim S: Immuno- and gene expression analysis of EGFR and Nestin during mice skin development. Tissue Cell 48: 274-281, 2016.

2. Mistriotis P and Andreadis ST: Hair follicle: A novel source of multipotent stem cells for tissue engineering and regenerative medicine. Tissue Eng Part B Rev 19: 265-278, 2013.

3. Takeo M, Lee W and Ito M: Wound healing and skin regeneration. Cold Spring Harb Perspect Med 5: a023267, 2015.

4. Myung PS, Takeo M, Ito M and Atit RP: Epithelial Wnt ligand secretion is required for adult hair follicle growth and regeneration. J Invest Dermatol 133: 31-41, 2013.

5. Hsu YC, Li L and Fuchs E: Emerging interactions between skin stem cells and their niches. Nat Med 20: 847-856, 2014.

6. Higgins CA, Chen JC, Cerise JE, Jahoda CA and Christiano AM: Microenvironmental reprogramming by three-dimensional culture enables dermal papilla cells to induce de novo human hair-follicle growth. Proc Natl Acad Sci USA 110: 19679-19688, 2013.

7. Biernaskie J, Paris M, Morozova O, Fagan BM, Marra M, Pevny L and Miller FD: SKPs derive from hair follicle precursors and exhibit properties of adult dermal stem cells. Cell Stem Cell 5: 610-623, 2009.

8. Sennett R and Rendl M: Mesenchymal-epithelial interactions during hair follicle morphogenesis and cycling. Semin Cell Dev Biol 23: 917-927, 2012.

9. Li L, Mignone J, Yang M, Matic M, Penman S, Enikolopov G and Hoffman RM: Nestin expression in hair follicle sheath progenitor cells. ProcNatlAcad Sci USA 100: 9958-9961, 2003.

10. Matsumura H, Mohri Y, Binh NT, Morinaga H, Fukuda M, Ito M, Kurata S, Hoeijmakers J and Nishimura EK: Hair follicle aging is driven by transepidermal elimination of stem cells via COL17A1 proteolysis. Science 35: 1aad4395, 2016.

11. Kandyba E and Kobielak K: Wnt7b is an important intrinsic regulator of hair follicle stem cell homeostasis and hair follicle cycling. Stem Cells 32: 886-901, 2014.

12. Hoffman RM: Nestin-expressing hair follicle-accessible pluripotent stem cells for nerve and spinal cord repair. Cells Tissues Organs 200: 42-47, 2014.

13. Müller-Röver S, Handjiski B, van der Veen C, Eichmüller S, Foitzik K, McKay IA, Stenn KS and Paus R: A comprehensive guide for the accurate classification of murine hair follicles in distinct hair cycle stages. J Invest Dermatol 117: 3-15, 2001.
14. Xie L, Zeng X, Hu J and Chen Q: Characterization of Nestin, a Selective marker for bone marrow derived mesenchymal stem cells. Stem Cells Int 2015: 762098, 2015.

15. Amoh Y, Li L, Yang M, Moossa AR, Katsuoka K, Penman S and Hoffman RM: Nascent blood vessels in the skin arise from nestin-expressing hair-follicle cells. Proc Natl Acad Sci USA 101: 13291-13295, 2004.

16. Uchugonova A, Cao W, Hoffman RM and Koenig K: Comparison of label-free and GFP multiphoton imaging of hair follicle-associated pluripotent (HAP) stem cells in mouse whiskers. Cell Cycle 14: 3430-3433, 2015.

17. Hu W, Lu H, Wang S, Yin W, Liu X, Dong L, Chiu R, Shen L, $\mathrm{Lu}$ WJ and Lan F: Suppression of Nestin reveals a critical role for p38-EGFR pathway in neural progenitor cell proliferation. Oncotarget 7: 87052-87063, 2016.

18. Park D, Xiang AP, Mao FF, Zhang L, Di CG, Liu XM, Shao Y, Ma BF, Lee JH, Ha KS, et al: Nestin is required for the proper self-renewal of neural stem cells. Stem Cells 28: 2162-2171, 2010.

19. Sahlgren CM, Mikhailov A, Vaittinen S, Pallari HM, Kalimo H, Pant HC and Eriksson JE: Cdk5 regulates the organization of Nestin and its association with p35. Mol Cell Biol 23: 5090-5106, 2003.

20. Isern J, Garcia-Garcia A, Martin AM, Arranz L, Martín-Pérez D, Torroja C, Sánchez-Cabo F and Méndez-Ferrer S: The neural crest is a source of mesenchymal stem cells with specialized hematopoietic stem cell niche function. Elife 3: e03696, 2014.

21. Kusumbe AP, Ramasamy SK, Itkin T, Mäe MA, Langen UH, Betsholtz C, Lapidot T and Adams RH: Age-dependent modulation of vascular niches for haematopoietic stem cells. Nature 532: 380-384, 2016

22. Ono N, Ono W, Mizoguchi T, Nagasawa T, Frenette PS and Kronenberg HM: Vasculature-associated cells expressing nestin in developing bones encompass early cells in the osteoblast and endothelial lineage. Dev Cell 29: 330-339, 2014.

23. Itkin T, Gur-Cohen S, Spencer JA, Schajnovitz A, Ramasamy SK, Kusumbe AP, Ledergor G, Jung Y, Milo I, Poulos MG, et al: Distinct bone marrow blood vessels differentially regulate haematopoiesis. Nature 532: 323-328, 2016.

24. Méndez-Ferrer S, Michurina TV, Ferraro F, Mazloom AR, Macarthur BD, Lira SA, Scadden DT, Ma'ayan A, Enikolopov GN and Frenette PS: Mesenchymal and haematopoietic stem cells form a unique bone marrow niche. Nature 466: 829-834, 2010. 